\title{
Estudo Comparativo das Sensilas Antenais de Operárias de Melipona scutellaris Latreille (Hymenoptera: Apidae) de Diferentes Altitudes*
}

\author{
Marcília Aparecida Nascimento1,2, Tânia Maria Fernandes Salomão² \& Gustavo Ferreira Martins²
}

1. Secretaria de Estado Educação de Mato Grosso - Educação Básica, e-mail: nascmarc@yahoo.com.br. 2. Universidade Federal de Viçosa, e-mail: fernands@ufv.br, gmartins@ufv.br (Autor para correspondência ${ }^{\bowtie}$ )

\section{EntomoBrasilis 6 (1): 64-67 (2013)}

Resumo. Sensilas são estruturas sensoriais presentes nas antenas dos insetos e são responsáveis pela higro- quimio-, termo- e mecanorrecepção. No presente trabalho, as sensilas presentes nos três flagelômeros (F) mais distais de Melipona scutellaris Latreille (Hymenopera: Apidae) coletadas em diferentes altitudes (200 e acima de 900m) foram avaliadas qualitativamente e quantitativamente. A identificação, contagem e medição das sensilas foram feitas a partir de imagens da superfície dorsal das antenas que foram obtidas com o auxílio de microscópio eletrônico de varredura. Foram observadas as sensilas placoide, basicônica, celocônica, ampulácea, campaniforme e tricoide reta e curvada. Comparativamente, o grupo localizado a 200m de altitude possui sensilas tricoides retas em maior quantidade nos três flagelômeros, enquanto que o grupo de altitudes acima de 9oom apresentou maior quantidade de sensilas tricoides curvadas no F9, sendo as mesmas maiores para o F10. Essas diferenças foram discutidas em função da localização geográfica dos diferentes grupos aqui estudados. Os resultados da investigação da estrutura antenal de M. scutellaris contribuem para um melhor entendimento da biologia dessa espécie.

Palavras-chave: Antena; Meliponini; Microscopia Eletrônica de Varredura; Órgão Sensorial.

\section{A Comparative Study of the Antennal Sensilla of Melipona scutellaris Latreille (Hymenoptera: Apidae) Workers From Different Altitudes}

Abstract. The sensilla are sensitive structures located in different insect parts including the antennae. They are responsible for the hygro-, chemo-, thermo- and mechanoreception. In the present work the antennal topography of two different populations of Melipona scutellaris Latreille (Hymenopera: Apidae) from different altitudes (200 and above 900m) were studied considering the last three distal antennal flagelomers (F). Images of the antennal surface were obtained with the aid of the scanning electron microscope (SEM). The SEM analysis showed that the sensilla placodea, basiconic, coeloconic, ampullacea, campaniform and trichodea are present in the dorsal surface of the M. scutellaris antennae. The number of sensilla trichodea of the straight subtype is greater in the last three flagelomers in individuals of $200 \mathrm{~m}$ altitude, while individuals located above $900 \mathrm{~m}$ altitude has a greater number of sensilla trichodea of the subtype curved in the F9 and they have greater length in individuals of 9oom altitude only in the F10. These differences were discussed according to the geographic location of the different groups studied here. This work represents the first assessment to antennal structure of M. scutellaris and contributes to a better understanding of the biology of this species.

Keywords: Antenna; Meliponini; Scanning Electron Microscopy; Sensitive Organ.

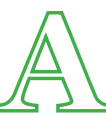
espécie de abelha "sem ferrão" Melipona scutellaris Latreille (Hymenopera: Apidae), conhecida popularmente como Uruçu do Nordeste ou Uruçu do Campo, originalmente, era encontrada desde a Amazônia até o estado da Bahia. Porém, dados atuais mostram que sua ocorrência restringe-se do estado da Bahia até o Rio Grande do Norte, sendo encontrada principalmente na região da Zona da Mata nordestina. Especificamente no estado da Bahia, $M$. scutellaris é encontrada nas regiões planálticas e serranas, com uma distribuição geográfica em diferentes altitudes (CAMARGo \& PEDRO 2007).

O meliponíneo M. scutellaris é um dos mais manipulados pelo homem, sendo uma das primeiras abelhas a ser domesticada pelos nativos e, atualmente, representa uma importante fonte de renda para os criadores da região nordeste pela produção de mel e de própolis (KeRR et al. 1996). Outra característica peculiar dessa espécie é o fato dela nidificar, principalmente, em ocos de árvores velhas de até $80 m$ de altura (Nunes et al. 2007).

Apesardasuaimportância do ponto devista econômicoeecológico, até o momento pouca atenção tem sido dada à biologia de $M$. scutellaris incluindo aspectos morfológicos das suas estruturas sensoriais. Um fator importante no estudo das interações insetoambiente são as estruturas sensoriais encontradas em diferentes partes do corpo, de forma que as informações sobre os recursos ambientais podem ser percebidas graças à presença das mesmas. Essas estruturas denominadas sensilas são encontradas em maior quantidade nas antenas e em menor número em outras partes da região da cabeça, como as maxilas e palpos labiais (BRUYNE \& BAKER 2008), nos tarsos e no aparelho ovipositor (GIANNAKAKIs \& FLETCHER 1985).

As sensilas são estruturas básicas da percepção dos estímulos ambientais cujas funções mecano-, quimio-, termo- e higrorreceptora estão relacionadas à sua morfologia. Apesar de morfologicamente simples, as sensilas apresentam uma grande variedade de funções, sendo capazes de receber estímulos luminosos, térmicos, químicos e mecânicos, bem como variações na umidade relativa do ar (revisado em Chapman 1998). Embora as funções das sensilas variem, sua composição celular é relativamente uniforme, sendo compostas por neurônios 
T bipolares, células tecogênicas, tricogênicas e tormogênicas (revisado em KeIL 1997).

A classificação, a abundância e a distribuição de sensilas nas antenas dependem da espécie de inseto (FAUchex et al. 2006). As sensilas antenais das abelhas, por exemplo, são classificadas como sendo dos tipos tricoide, basicônica, celocônica, campaniforme, placoidea e ampulácea (CRUZ-LANDIM 2009). Além de variar quanto à espécie, a quantidade e o tipo de sensilas também podem variar entre indivíduos de uma mesma espécie dependendo da sua distribuição geográfica (EsteBAn et al. 2005). Nesse contexto, no presente estudo foram avaliados os tipos, a distribuição e a quantidade de sensilas tricoides retas e curvadas das extremidades antenais de duas populações de $M$. scutellaris de diferentes altitudes.

\section{MATERIAL E MÉTODOS}

Operárias de $M$. scutellaris foram coletadas diretamente nas entradas dos ninhos em regiões de altitudes diferentes no estado da Bahia. Foi utilizado um indivíduo por ninho, sendo 10 indivíduos oriundos da região do município de Cruz das Almas ( $12^{\circ} 39^{\prime} \mathrm{S}$ e $\left.39^{\circ} \mathrm{O} 6^{\prime} \mathrm{W}, 200 \mathrm{~m}\right)$, localizado a $200 \mathrm{~m}$ de altitude, seis indivíduos oriundos de Maracás (13 $\left.{ }^{\circ} 26^{\prime} \mathrm{S} 40^{\circ} 25^{\prime} \mathrm{W}, 915 \mathrm{~m}\right)$ e quatro do Morro do Chapéu ( $\left.11^{\circ} 33^{\prime} \mathrm{S} 41^{\circ} \mathrm{O} 9^{\prime} \mathrm{W}, 1.100 \mathrm{~m}\right)$. Os indivíduos coletados em Cruz das Almas foram incluídos no grupo denominado grupo de 200m de altitude e os de Maracás e Morro no grupo denominado grupo acima de $900 \mathrm{~m}$ de altitude.

Foi retirada uma antena de cada indivíduo do lado direito ou esquerdo, as mesmas foram lavadas com solução fisiológica, seguido da desidratação em solução de acetona em concentrações crescentes (50\%-100\%) por 7 minutos em cada concentração. As amostras foram submetidas à secagem no aparelho de ponto crítico com $\mathrm{CO}_{2}$ e posteriormente montadas em suportes de alumínio, metalizadas com ouro e fotografadas em microscópio eletrônico de varredura (MEV) modelo LEO VP1430 no Núcleo de Microscopia e Microanálise da Universidade Federal de Viçosa.

Os dados para a identificação e a quantificação das sensilas antenais foram obtidos diretamente das elétron-micrografias, considerando apenas os três últimos flagelômeros (mais distais) por concentrarem a maior parte das sensilas antenais (CRUZLANDIM 2009). Para a quantificação, foram consideradas apenas as sensilas tricoides (quimiorreceptoras) de extremidades reta e curvada por estas estarem em maior quantidade e por serem de fácil visualização e identificação, comparativamente às demais sensilas. Os números de sensilas foram obtidos pela contagem em uma área quadrada de tamanho fixo de 960 pixels ao longo de cada flagelômero utilizando o programa Adobe Photoshop 7.0. Também foi medido o comprimento $(\mu \mathrm{m})$ das sensilas tricoides curvadas. Todas as imagens analisadas possuíam 72 pixels/ polegada. As medidas do comprimento $(\mu \mathrm{m})$ foram feitas com o auxílio do programa Image ProPlus 4.5.

As comparações do número e comprimento das sensilas tricoides entre os dois grupos estudados foram feitas com o auxílio do programa SAEG (Sistema para Análises Estatísticas). Os dados obtidos foram submetidos à análise de variância (ANOVA) e ao teste F com nível de significância de 5\%.

\section{RESULTADOS}

As antenas de operárias de $M$. scutellaris são do tipo geniculadas, consistem de 10 flagelômeros conectados ao escapo por meio de um pedicelo e apresentam sensilas distribuídas ao longo de todo seu comprimento (Figura 1A). Em relação aos tipos de sensilas encontradas nos três últimos flagelômeros antenais de M. scutellaris foram identificadas as do tipo tricoide, placoide, basicônica, celocônica, ampulácea e campaniforme. No caso das sensilas tricoides, as mesmas exibem tamanhos e curvaturas diferentes, sendo as do subtipo curvada mais abundantes no flagelômero mais distal (Figura 1B).
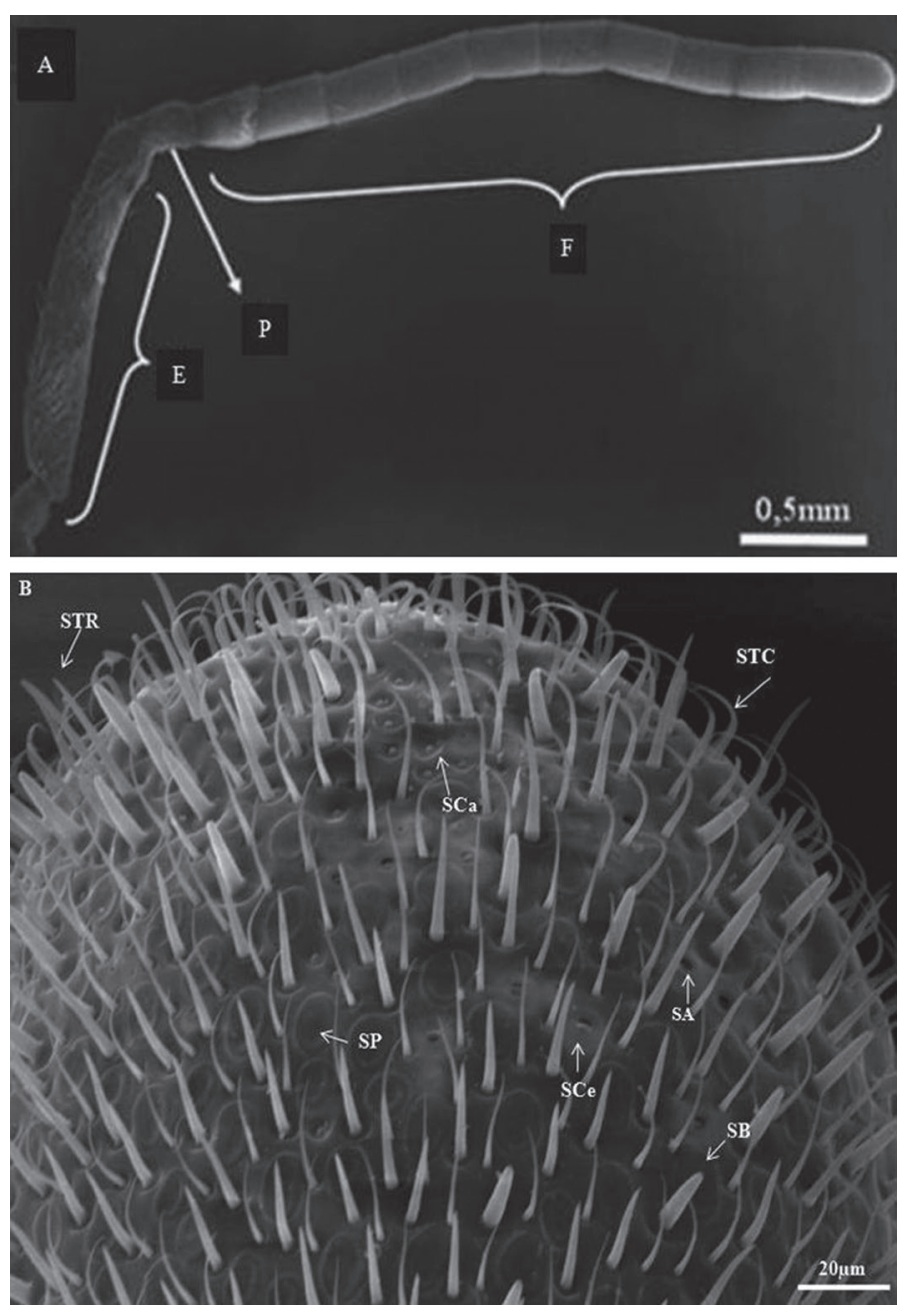

Figura 1. Micrografias eletrônicas de varredura mostrando aspectos gerais e detalhados da superfície dorsal da antena de operárias adultas de M. scutellaris. (A) Microgafia mostrando aspectos gerais da antena com suas principais partes: E- escapo, P- pedicelo, e F- flagelômeros. (B) Micrografia evidenciando diferentes tipos de sensilas: STR: sensila tricóide de extremidade reta; STC: sensila tricóide de extremidade curvada; SCa: sensilas campaniforme; SB: sensila basicônica; SA: sensila ampulácea; SP: sensila placoides e Sce: sensila celocônica.

Os números desensilas tricoides deextremidaderetasãodiferentes entre os dois grupos de $M$. scutellaris estudados, considerando os três flagelômeros (F8, F9 e F10) analisados [F8 (F=0,00007, $\mathrm{P}<0,05) ; \mathrm{F} 9(\mathrm{~F}=0,00470, \mathrm{P}<0,05) ; \mathrm{F} 10(\mathrm{~F}=0,01648, \mathrm{P}<0,05)]$ (Figura 2A). No grupo de 200m de altitude, o número de sensilas tricoides de extremidade reta é maior do que no grupo acima de $900 m$ de altitude, sendo as médias dos números dessas sensilas no grupo de $200 \mathrm{~m}$ de $\mathrm{F} 8=62,9 \pm 1,05, \mathrm{~F} 9=64,6 \pm 0,92$ e $\mathrm{F} 10=$ $62,4 \pm 1,17$; enquanto as médias do grupo de acima de $900 \mathrm{~m}$ de altitude são de $\mathrm{F} 8=57,7 \pm 1,46, \mathrm{~F} 9=59,5 \pm 1,29$ e $\mathrm{F} 10=58,1 \pm 1,10$ (Figura 2A). O grupo de $200 \mathrm{~m}$ de altitude possui um menor número de sensilas tricoides curvadas no F9, com uma média de $16,3 \pm 0,95$ sensilas para $200 m$ e $20,0 \pm 1.35$ para acima de $900 m$ de altitude $(\mathrm{F}=0,03733, \mathrm{P}<0,05)$ (Figura 2B). A sensila curvada apresenta menor comprimento no grupo de $200 \mathrm{~m}$ de altitude apenas no F10, medindo em média $15.0 \pm 0.40 \mu \mathrm{m}$, enquanto no grupo acima de $900 \mathrm{~m}$ a média foi de $15,5 \pm 0,43 \mu \mathrm{m}(\mathrm{F}=0,00071$ $\mathrm{P}<0$,05) (Figura 2C).

\section{DISCUSSÃO}

A análise por MEV possibilitou identificar os tipos de sensilas presentes em $M$. scutellaris, sendo elas dos tipos basicônica, placoide, celocônica, tricoide, campaniforme e ampulácea. Esses resultados corroboram observações feitas em antenas de outras espécies de abelhas, sendo esses seis tipos de sensilas observados em Melipona quadrifasciata Lepeletier e Apis melifera Linnaeus (Hymenoptera: Apidae) (Frasnelli et at. 2010). Esses mesmos 
tipos de sensilas foram também observados em formigas das espécies Solenopsis invicta Buren (Hymenoptera: Formicidae) (RENTHAL et al. 2003) e Dinoponera lucida Emery (Hymenoptera: Formicidae) (MARQUES-Silva et al. 2006), mostrando que esses tipos de sensilas são conservados entre os Hymenoptera sociais.
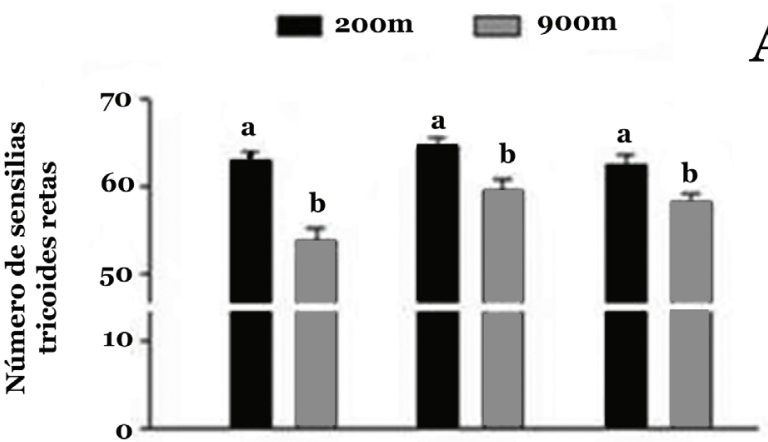

B
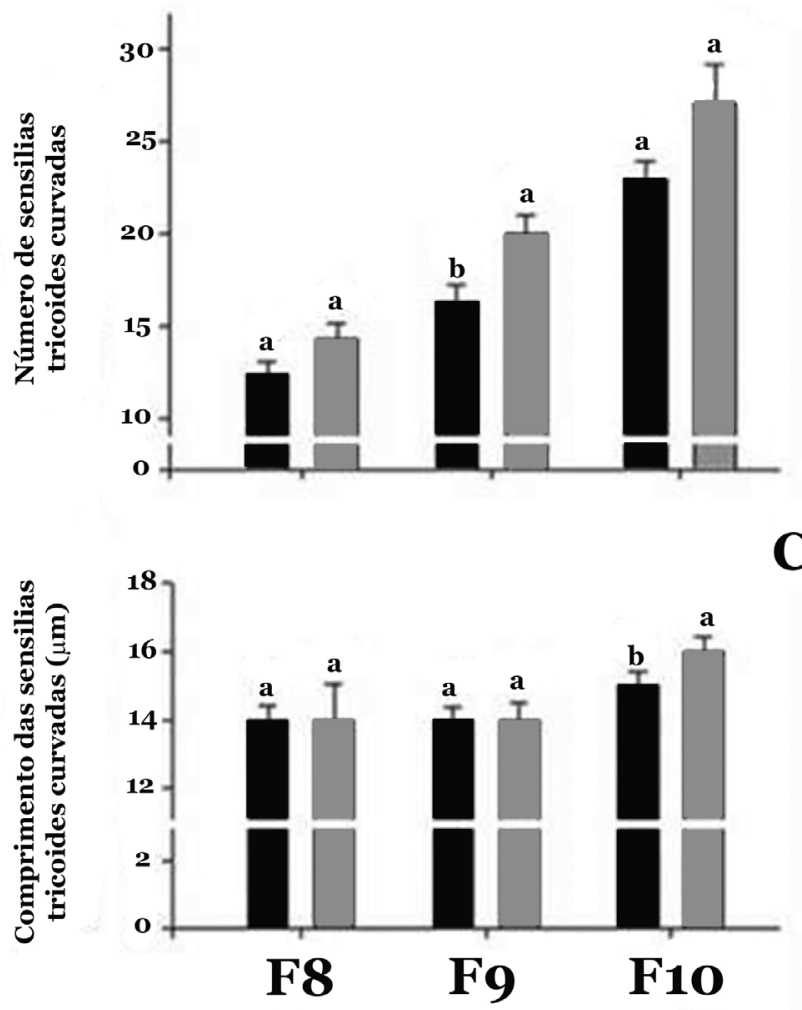

Figura 2. Número e tamanho das sensilas tricoides da superfície dorsal dos flagelômeros F8, F9 e F10 da antena de operárias adultas de M. scutellaris de diferentes altitudes. As barras de cor preta representam população de 200m de altitude, enquanto que as de cor cinza representam população localizada acima de $900 m$ de altitude. As letras a e b representam diferenças significativas a 5\%. (A) Número das sensilas tricoides de extremidades retas; (B) Número e (C) tamanho das sensilas tricoides de extremidades curvadas.

As sensilas placoides são encontradas ao longo de toda superfície da antena de $M$. scutellaris e pela simples inspeção visual, foi possível notar que a quantidade delas aumenta à medida que se aproxima da extremidade do apêndice. A função dessas sensilas é descrita como sendo olfativa em alguns Hymenoptera (OcHIENG et al. 2000; Spaethe et al. 2007) e foi estudada em detalhes na espécie de abelha Bombus terrestris Linnaeus (Hymenoptera: Apidae), mostrando sua importância para a percepção de odores. Nesse caso, dentro de uma mesma colônia, os indivíduos com maior densidade dessas sensilas exibiam melhor capacidade de reposta a odores do que os indivíduos com antenas pequenas e menor densidade de sensilas (SPAETHE et al. 2007). Apesar da função de quimiorrecepção ainda não ter sido confirmada para $M$. scutellaris, o fato de essas sensilas estarem mais abundantes na extremidade antenal sugere que as mesmas podem atuar como quimiorreceptoras.
Outros tipos de sensilas com funções relacionadas à percepção de estímulos, como as ampuláceas, celocônicas e campaniformes, estão também concentradas na extremidade do último flagelômero (F10) antenal de $M$. scutellaris, porém, em quantidades relativamente baixas em comparação às tricoides e placoides. Embora em menor quantidade, essa localização na extremidade antenal também poderia facilitar a percepção de estímulos externos relacionados a variações de umidade e de temperatura pelas sensilas ampuláceas, celocônicas e campaniformes durante o forrageio pelo fato da extremidade antenal estar mais exposta (Silva et al. 2011). Apesar de ter sido observado maior quantidade de sensilas tricoides e placoides em relação às sensilas basicônicas, essas últimas também são consideradas importantes quimiorreceptoras mesmo estando em menor quantidade (MARTINI \& SCHMIDT 1984).

As variações morfométricas observadas nas sensilas tricoides de extremidade reta e de extremidade curvada entre os grupos avaliados não são exclusividade delas considerando populações de $M$. scutellaris de diferentes altitudes. NunEs et al. (2007) mostraram que o tamanho das asas das operárias dessa espécie também variou de acordo com a altitude. Esses estudos da morfometria das asas realizadas por NunEs et al. (2007) separaram M. scutellaris em três grupos distintos de indivíduos conforme a altitude: grupos localizados a $1.100 \mathrm{~m}$, de o a $560 \mathrm{~m}$ e de 600 a $964 \mathrm{~m}$. Outras diferenças morfológicas notadas nessa espécie foram observadas por esses autores, onde os espécimes coletados próximos ao nível do mar apresentavam abdômen relativamente grande e escuro, enquanto que à medida que aumentava a altitude, o tamanho do abdômen diminuía gradativamente e as abelhas apresentavam coloração mais clara e maior pilosidade no tórax. Portanto, pode-se concluir que em $M$. scutellaris o fenótipo das operárias depende da altitude em que eles são encontrados e isso não está restrito apenas ao tamanho do corpo ou da asa, mas também em características microanatômicas, incluindo o tamanho de sensilas.

A percepção da umidade de modo mais acurado permite ao inseto determinar as condições meteorológicas mais apropriadas para o forrageamento ou aliado ao aparato olfativo, determinar o melhor local e condições do alimento a ser coletado (KLEINEIDAM \& TAUTZ 1996; WeIDENMULLer et al. 2002). Considerando isso, a existência de um maior número de sensilas de extremidade reta nos três flagelômeros das abelhas do grupo de $200 \mathrm{~m}$ pode representar uma adaptação a fatores como a umidade do ar relativamente alta e uma menor incidência de ventos, que poderiam dificultar a difusão de odores. Nesse sentido, uma maior quantidade desse tipo de sensila nesse grupo possibilitaria uma melhor percepção das condições ambientais em altitudes mais baixas, mais úmidas e com mais ventos. Por outro lado, as abelhas de altitude mais elevada (>900m) e com temperatura e índice pluviométrico menores em relação à altitude menor, apresentam sensilas tricoides curvadas maiores em maior número (pelo menos em F9) em relação às observadas nos indivíduos do grupo de $200 m$. Essa diferença nos indivíduos acima de 90om sugere que isso possa ser uma adaptação dos mesmos que vivem em altitudes relativamente maiores. Porém, até o momento, o papel de órgãos sensoriais mais desenvolvidos para esse tipo de ambiente é desconhecido.

Embora os mesmos tipos de sensilas tenham sido encontrados nas antenas das M. scutellaris dos dois grupos avaliados, diferenças significativas foram observadas no número e no comprimento dos dois morfotipos de sensilas tricoides. Esses resultados mostram que a análise de sensilas nessas abelhas pode constituir em uma ferramenta importante para o entendimento da interação desses insetos com o ambiente. Além disso, dentre os fatores abióticos determinantes das diferenças fenotípicas, sugerimos que a altitude e fatores associados como umidade, pluviosidade e temperatura podem estar estreitamente relacionados com as diferenças observadas entre os dois grupos de M. scutellaris estudados. Os resultados obtidos nesse estudo mostram que a 
altitude e fatores associados à mesma podem estar contribuindo com as diferenças no tamanho e número de sensilas tricoides observadas entre os grupos de $M$. scutellaris estudados.

\section{AGRADECIMENTOS}

À Secretaria de Educação do Estado de Mato Grosso (SEDUC/ MT), à FAPEMIG, à Universidade Federal de Viçosa (UFV) e ao CNPq pelo apoio financeiro e ao Núcleo de Microscopia e Microanálise pelo apoio técnico.

\section{REFERÊNCIAS}

Bruyne, M. \& T.C. Baker, 2008. Odor detection in insects: volatile codes. Journal of Chemical Ecology, 34: 882-897.

Camargo, J.M.F \& S.R.M. Pedro, 2007. Meliponini Lepeletier, 1836, p. 272-578. In: Moure, J.S., Urban, D. \& G.A.R. Melo (Eds.). Catalogue of Bees (Hymenoptera, Apoidea) in the Neotropical Region. Curitiba, Sociedade Brasileira de Entomologia, 1058p

Chapman, R.F., 1998. The Insects Structure and Function. Cambridge, Cambridge University Press, 770p.

Cruz-Landim, C., 2009. Abelhas: Morfologia e Função de Sistemas. São Paulo, Unesp. 408p.

Esteban, L.V.M. Angulo, M.D. Feliciangeli \& S. Catalá, 2005. Analysis of antenal sensilla patterns of Rhodnius prolixus from Colombia and Venezuela. Memórias do Instituto Oswaldo Cruz, 100: 909-914.

Faucheux, M.J., N.P. Kristensen \& S.H. Yen, 2006. The antennae of neopseustid moths: morphology and phylogenetic implications with special reference to the sensilla (Insecta; Lepidoptera; Neopseustidae). Zoologischer Anzeiger, 245: 131-142.

Frasnelli, E., G. Anfora, F. Trona, F. Tessarolo \& G. Vallortigara, 2010. Morpho-functional asymmetry of the olfactory receptors of the honeybee (Apis mellifera). Behavioural Brain Research, 209: 221-225.

Giannakakis, A. \& B.S. Fletcher, 1985. Morphology and distribution of antennal sensilla of Dacus tryoni (Froggatt) (Diptera, Tephritidae). Journal of the Australian of Entomological Society, 24: 31-35.

Keil T.A., 1997. Comparative morphogenesis of sensila: a review. International Journal of Morphology \& Embryology, 26: 151160.
Kerr, W.E., G.A. Carvalho \& V.A. Nascimento, 1996. Abelha Uruçu. Biologia, Manejo e Conservação. Belo Horizonte: Acangaú, 144p.

Kleineidam, C. \& J. Tautz, 1996. Perception of carbon dioxide and other "air-condition" parameters in the leaf cutting ant Atta cephalotes. Naturwissenschaften, 83: 566-568.

Marques-Silva S., C.P. Matiello-Guss, J.H.C. Delabie, C.S.F. Mariano, J.C. Zanuncio \& J.E. Serrão, 2006. Sensilla and secretory glands in the antennae of a primitive ant: Dinoponera lucida (Formicidae: Ponerinae). Microscroscopy Research and Techinique, 69: 885-890.

Martini, R. \& K. Schmidt, 1984. Ultrastructure and early development of the pore plate sensilla of the Gymnomerus laevipes (Shuckard) (Vespoidea, Eumenidade). Protoplasma, 119: 197-211.

Nunes, L.A., M.F.F.C. Pinto, P. Carneiro, D.G. Pereira \& A.M. Waldschmidt, 2007. Divergência genética em Melipona scutellaris Latreille (Hymenoptera: Apidae) com base em caracteres morfológicos. Bioscience Journal, 23: 1-9.

Ochieng, S.A., K.C. Park, J.W.Zhu \& T.C. Baker, 200o. Functional morphology of antennal chemoreceptors of the parasitoid Microplitis croceipes (Hymenoptera: Braconidae). Arthropod Structure and Development, 29: 232-240.

Renthal, R., D. Velasquez, D. Olmos, J. Hampton \& W.P. Wergin, 2003. Structure and distribution of antennal sensilla of the red imported fire ant. Micron, 34: 405-413.

Silva, M.D., M. Ramalho \& J.F. Rosa, 2011. Por que Melipona scutellaris (Hymenoptera, Apidae) forrageia sob alta umidade relativa do ar? Iheringia, Série Zoologia, 101: 131-134.

Spaethe, J., A. Brockmann, C. Halbig \& J. Tautz, 2007. Size determines antennal sensitivity and behavioral threshold to odors in bumblebee workers. Naturwissenschaften, 97: 733739 .

Weidenmuller, A., C. Kleineidam \& J. Tautz, 2002. Collective control of nest climate parameters in bumblebee colonies. Animal Behaviour, 63: 1065-1071.

\section{Recebido em: 25/05/2012}

Aceito em: 12/12/2012

\section{Como citar este artigo:}

Nascimento, M.A., T.M.F. Salomão \& G.F. Martins, 2013. Estudo Comparativo das Sensilas Antenais de Operárias de Melipona scutellaris Latreille (Hymenoptera: Apidae) de Diferentes Altitudes. EntomoBrasilis, 6(1): 64-67.

Acessível em: http://www.periodico.ebras.bio.br/ojs/index.php/ebras/article/view/252. doi:10.12741/ebrasilis.v6i1.252
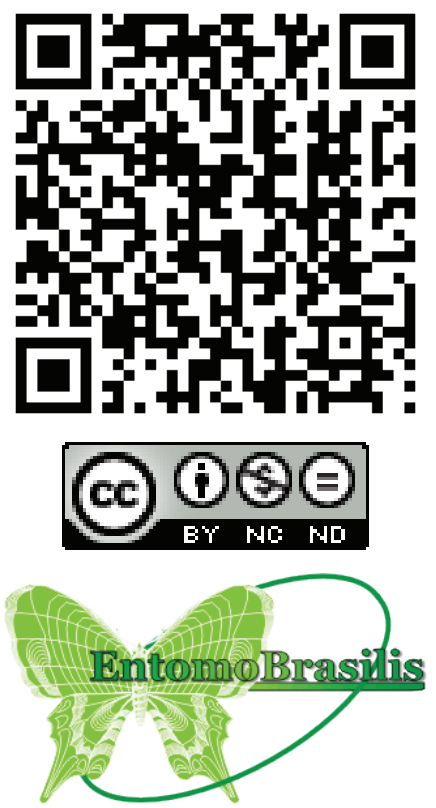